\title{
Cadaveric and Ultrasonographic Validation of Needling Placement in the Cervical Multifidus Muscle
}

\author{
César Fernández-de-las-Peñas, PT, PhD, ${ }^{\text {a, b }}$ Juan A. Mesa-jiménez, PT, MSc, ${ }^{c, d}$ \\ Jose A. Paredes-Mancilla, MD, PhD, ${ }^{e}$ Shane L. Koppenhaver, PT, PhD, ${ }^{f}$ and \\ Samuel Fernández-Carnero, PT, MSc ${ }^{\mathrm{g}}$
}

\begin{abstract}
Objective: The aim of this study was to determine if a needle is able to reach the cervical multifidus during the application of dry needling or acupuncture.

Methods: Dry needling and ultrasound imaging of cervical multifidi was conducted on 5 patients (age: $32 \pm 5$ years) with mechanical neck pain and on 2 fresh cadavers (age: $64 \pm 1$ years). Dry needling was done using a needle of $40 \mathrm{~mm}$ in length inserted perpendicular to the skin about $1 \mathrm{~cm}$ lateral to the spinous process at $\mathrm{C} 3-\mathrm{C} 4$. The needle was advanced from a posterior to anterior direction into the cervical multifidus with a slight inferior-medial angle (approximately $10^{\circ}$ ) to reach the vertebra lamina. For the cadaveric study, the multifidus was isolated by carefully resecting the superficial posterior cervical muscles: trapezius, splenius, and semispinalis. For the ultrasonographic study, a convex transducer was placed transversely over C3-C4 after the insertion of the needle into the muscle.

Results: The results of both the cadaveric and ultrasonic studies found that the needle does pierce the cervical multifidus muscle during insertion and that the tip of the needle rests properly against the vertebral laminae, thereby guarding the sensitive underlying spinal structures from damage.

Conclusion: This anatomical and ultrasound imaging study supports that dry needling of the cervical multifidus could be conducted clinically. (J Manipulative Physiol Ther 2017;xx:0-6)

Key Indexing Terms: Spine; Cervical Vertebrae; Neck Muscles; Ultrasonography
\end{abstract}

\footnotetext{
a Department of Physical Therapy, Occupational Therapy, Physical Medicine and Rehabilitation of Universidad Rey Juan Carlos, Alcorcón, Spain.

${ }^{\mathrm{b}}$ Cátedra de Investigación y Docencia en Fisioterapia: Terapia Manual y Punción Seca, Universidad Rey Juan Carlos, Alcorcón, Madrid, Spain.

${ }^{c}$ Department of Physical Therapy, Universidad San-Pablo CEU, Madrid, Spain.

${ }^{\mathrm{d}}$ Máster Oficial en Dolor Orofacial y Disfunción CráneoMandibular, Universidad San-Pablo CEU, Madrid, Spain.

e Department of Anatomy and Medicine, Universidad San-Pablo CEU, Madrid, Spain.

${ }^{\mathrm{f}}$ US Army-Baylor University Doctoral Program in Physical Therapy, San Antonio, Texas.

${ }^{g}$ Department of Physical Therapy, Universidad Francisco de Victoria, Madrid, Spain.

Corresponding author: César Fernández-de-las-Peñas, PT, PhD, Departamento de Fisioterapia, Facultad de Ciencias de la Salud, Universidad Rey Juan Carlos, Avenida de Atenas s/n, 28922 Alcorcón, Madrid, Spain. (e-mail: cesar.fernandez@urjc.es).

Paper submitted December 23, 2015; in revised form January

21, 2016; accepted March 30, 2016.

0161-4754

(C) 2017 by National University of Health Sciences.

http://dx.doi.org/10.1016/j.jmpt.2017.03.002
}

\section{INTRODUCTION}

Neck pain represents a health care problem whose lifetime and point prevalence is almost as great as low back pain. The economic burden of cervical disorders involves high annual compensation costs. A recent study found a global point prevalence of neck pain of $4.9 \%$ and disability-adjusted life years around 33.6 million. ${ }^{1}$ In this study, neck pain ranked as the fourth highest condition in terms of disability as measured by years of life lived with disability and as the 21 st in terms of overall burden. ${ }^{1}$

Better understanding of management strategies is needed for this condition. ${ }^{2}$ In the last decade, the cervical muscles, particularly deep cervical flexors and extensors, have been proposed to have a relevant role in neck pain conditions. In fact, it has been suggested that cervical extensors are as important as deep cervical flexors for proper treatment of individuals with neck pain. ${ }^{3}$ This hypothesis is supported by the fact that individuals with neck disorders exhibit 
different morphologic changes in this muscle. For example, fatty tissue infiltration of multifidus muscles has been identified in patients with whiplash-related neck pain. ${ }^{4}$ Furthermore, patients with mechanical neck pain have reduced activation of the multifidus and semispinalis cervicis muscles ${ }^{5}$ or decreased cross-sectional areas of the cervical multifidus. ${ }^{6}$ These morphologic changes in the deep cervical extensors are proposed to be contributing or perpetuating factors for neck pain disorders. ${ }^{7}$

Therefore, treatment approaches targeting the deep extensor muscles are clearly advocated in the management of individuals with neck pain. Manual therapy has been reported to benefit individuals with neck pain. ${ }^{8}$ However, palpation, and therefore direct manual treatment of cervical multifidus muscles, is not possible because the multifidus is the deepest muscle running over the lamina of the vertebra and is directly attached to the capsules of the cervical zygapophyseal joints. ${ }^{9}$ Because of this, exercise is the main therapeutic approached proposed for the management of the deep cervical extensors. ${ }^{7}$ Dry needling (an invasive procedure in which a stainless-steel needle is introduced into the muscle) has been also advocated as a possible therapeutic tool for the management of multifidus muscles. ${ }^{10}$ Nevertheless, evidence on dry needling of multifidus muscles is restricted to some case reports that documented a positive clinical effect of dry needling of the lumbar ${ }^{11}$ and thoracic ${ }^{12}$ multifidus musculature. A recent study noted that patients with low back pain who reported clinical improvements also exhibited larger increases in lumbar multifidus muscle contraction 1 week after dry needling compared with those patients who did not achieve a positive clinical response. ${ }^{13}$ In the cervical spine, it has been proposed that the inclusion of dry needling of the cervical multifidus muscle was effective for treating myofascial pain in elderly patients. ${ }^{14}$

Although dry needling may be able to adequately affect the cervical multifidus muscle, it is also important to ensure that commonly used procedures are clinically safe. The technique generally advocated for treatment of the cervical multifidus muscle uses an approach that goes all the way to the cervical laminae, which serves to protect the underlying spinal cord. ${ }^{10}$ However, at least 1 case report has documented serious spinal injury to the deep cervical spinal after dry needling treatment. ${ }^{15}$

The clinical relevance of multifidus muscles for spinal pain makes it worthwhile to develop the necessary skills for invasive treatment such as dry needling. Therefore, it is important to determine if clinicians are able to properly reach the cervical multifidus muscle with a needle. To the best of the authors' knowledge, no anatomic study has investigated if a needle (such as used in dry needling or acupuncture) is able to reach the cervical multifidus muscle. Therefore, the aim of the present study was to determine with fresh cadavers and ultrasound imaging if a needle is able to properly reach the cervical multifidus muscle during a needling treatment.

\section{MetHODS}

This study was approved by the human research committee of the Universidad San Pablo CEU (Madrid, Spain) All human participants signed an informed consent before their inclusion.

\section{Dry Needling of the Cervical Multifidus Muscle}

The needle approximation is based on anatomic characteristics of the cervical multifidus muscle because this muscle is not directly palpable. The study was conducted at the C3-C4 cervical level. For this approach, sterile stainless steel needles $40 \mathrm{~mm}$ in length and $0.32 \mathrm{~mm}$ caliber with a plastic cylindrical guide were used. A "clean technique," which includes washing hands, wearing clean latex-free exam gloves, and cleaning the area skin with an alcohol swab before treatment, was used. ${ }^{16,17}$ The needling technique was identical in both the cadavers and the live patients.

With the patient or cadaver lying prone, the needle was inserted perpendicular to the skin about $1 \mathrm{~cm}$ lateral to the spinous process at the C3-C4 level. The needle was then advanced from a posterior to anterior direction into the multifidus muscle with a slight inferior-medial angle (approximately $10^{\circ}$ ) to reach the lamina of the cervical vertebra. Figure 1 shows the application of the needling approach on a real patient, whereas Figure 2 shows insertion and preparation on a fresh cadaver.

\section{Anatomic Study}

Two heads, including the cervical spine, were removed from two fresh cadavers aged 64 years, which were preserved in a mixture of formalin, alcohol, and Lysoformin (Medilab, Madrid, Spain). Anatomic dissection was conducted by an anatomist with more than 20 years of experience at the Anatomy Laboratory of Universidad San Pablo CEU, Madrid, Spain.

First, needling of the cervical multifidus muscle was performed on the fresh cadaver as described (Fig 2). The needle was left in during anatomic dissection. The skin and superficial fascia of the posterior part of the cervical spine muscles were removed. The multifidus muscle was isolated by carefully and progressively resecting the more superficial posterior muscles of the cervical region: the trapezius, splenius, and semispinalis. ${ }^{18}$ During the removal of the superficial posterior cervical muscles, care was taken to ensure that dissection did not damage the surrounding tissues in the aim to expose the multifidus musculature.

\section{Ultrasound Imaging Study}

Ultrasound imaging assessment was conducted during dry needling of 5 patients with mechanical neck pain. Participants were volunteers recruited by announcements in 


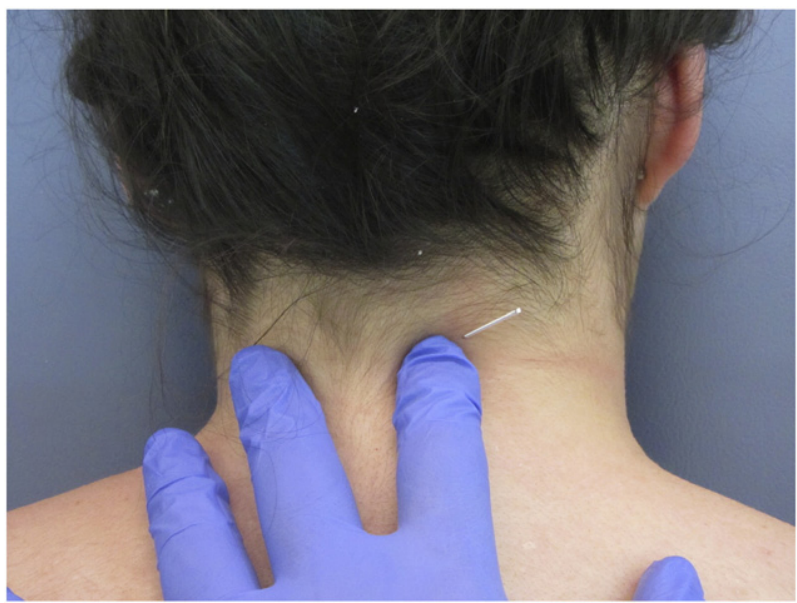

Fig I. Dry needling of the cervical multifidus muscle on a patient.

a local newspaper. Mechanical neck pain was defined in this study as generalized neck and shoulder pain with symptoms provoked by neck postures, neck movement, or palpation of the cervical musculature. Participants were excluded if they had a history of any of the following: (1) whiplash injury; (2) cervical surgery; (3) cervical radiculopathy or myelopathy; or (4) diagnosis of fibromyalgia syndrome.
A MINDRAY M9 ultrasound scanner was used with a 5-MHz convex (50-mm footprint) transducer (Shenzhen Mindray Co. Ltd, Shenzhen, China). The patients were positioned prone on the examination table with both upper extremities along the side of the body. Their faces rested in the hole of the head section of the table, permitting the head and neck to remain in a neutral position. The spinous process at the $\mathrm{C} 4$ level was identified by palpation and

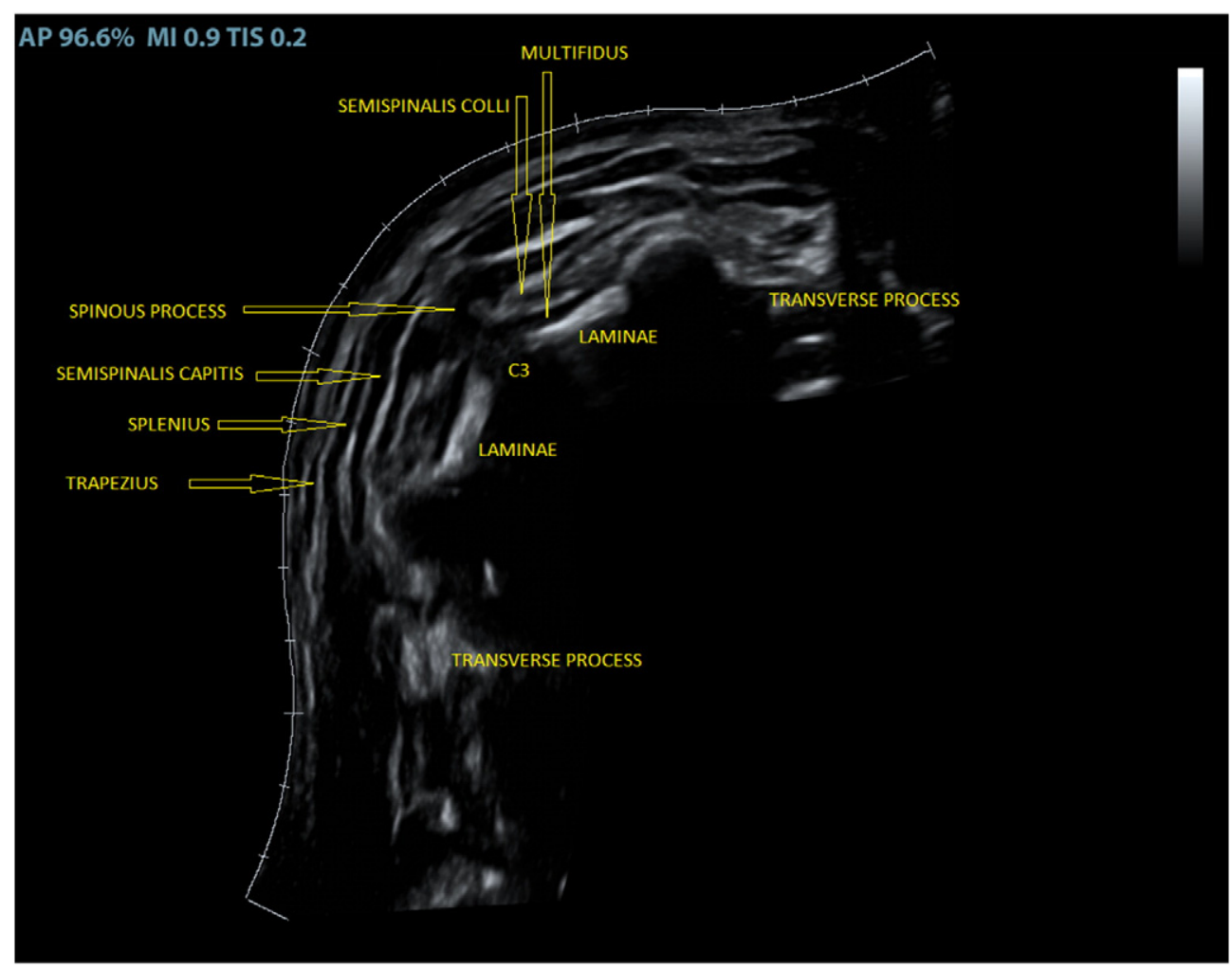

Fig 2. Panoramic ultrasound image showing the left and right deep posterior neck muscles of the targeted level. Color version of figure available online. 


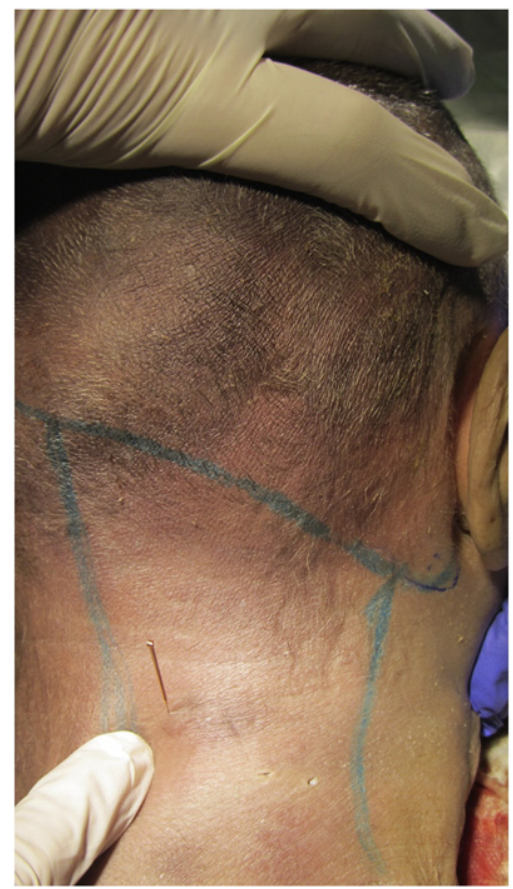

Fig 3. Dry needling of the cervical multifidus muscle on a fresh cadaver.

marked with a pen. The convex transducer was placed transversely in the midline over $\mathrm{C} 3-\mathrm{C} 4$, and the spinous process and laminae were identified. The vertebral laminae were used as a consistent landmark to identify the deep border of the muscle. Figure 3 shows panoramic ultrasound imaging of the targeted level. This procedure has been used in previous studies and has produced acceptable intratester and intertester reliability. ${ }^{19,20}$ First, the needling insertion of the cervical multifidus was performed, and the ultrasound study was conducted with the needle inserted into the cervical spine of the patient.

\section{RESULTS}

Needling and ultrasound imaging of multifidus musculature was conducted on 5 participants, 3 men and 2 women, mean age $32 \pm 5$ years, with neck pain; and on 2 fresh cadavers, mean age $64 \pm 1$ years. After removing the superficial posterior neck muscles in the cadaveric study, Figure 4 shows that the tip of the needle pierced the belly of the cervical multifidus muscle. Additionally, the tip of the needle was directly against the broad base of the vertebral laminae. Similarly, Figure 5 depicts a representative ultrasound image showing how the needle properly reached the cervical multifidus over the laminae. This was found to occur in all 5 live patients.

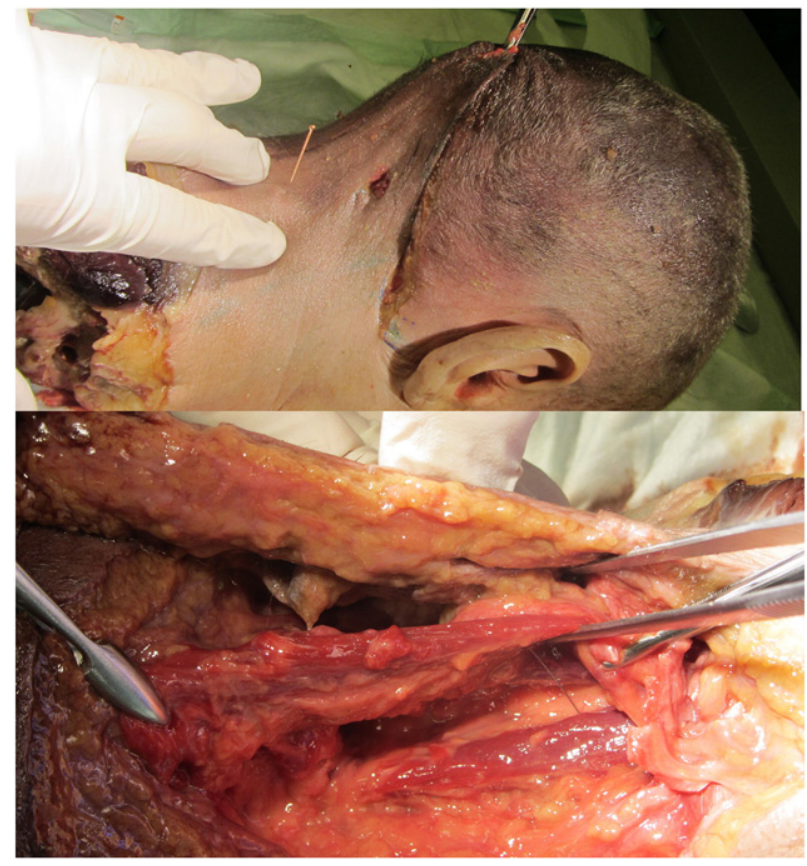

Fig 4. Dissection of a fresh cadaver's head showing that the needle properly reached the cervical multifidus muscle.

\section{DISCUSSION}

It is currently known that individuals with musculoskeletal pain disorders of the neck exhibit morphologic changes in the cervical multifidus. ${ }^{7}$ Although therapeutic exercise is proposed as the primary treatment targeting the musculature, other therapies, including dry needling, are also advocated. ${ }^{10}$ However, the anatomic location of the cervical multifidus creates doubts about proper access to the muscle. Our cadaveric and ultrasound imaging study supports that needling of cervical multifidus muscle may be accurately conducted in common clinical practice. The results of both the cadaveric and ultrasonic study found that the needle does pierce the cervical multifidus muscle during insertion and that the tip of the needle rests properly against the vertebral laminae, thereby guarding the sensitive underlying spinal structures from damage.

The results from the present study have several potential implications for clinical practice. First, because the cervical multifidus is not accessible to manual palpation, dry needling may represent a proper therapeutic approach for this muscle. Therefore, clinicians can use the proposed approach to properly reach the cervical multifidus with the needle to inactivate trigger points in participants with neck pain. In fact, preliminary evidence suggests that dry needling of the cervical multifidus muscle is effective for the management of older individuals with neck pain. ${ }^{14}$ Another clinical implication may be related to activation of 


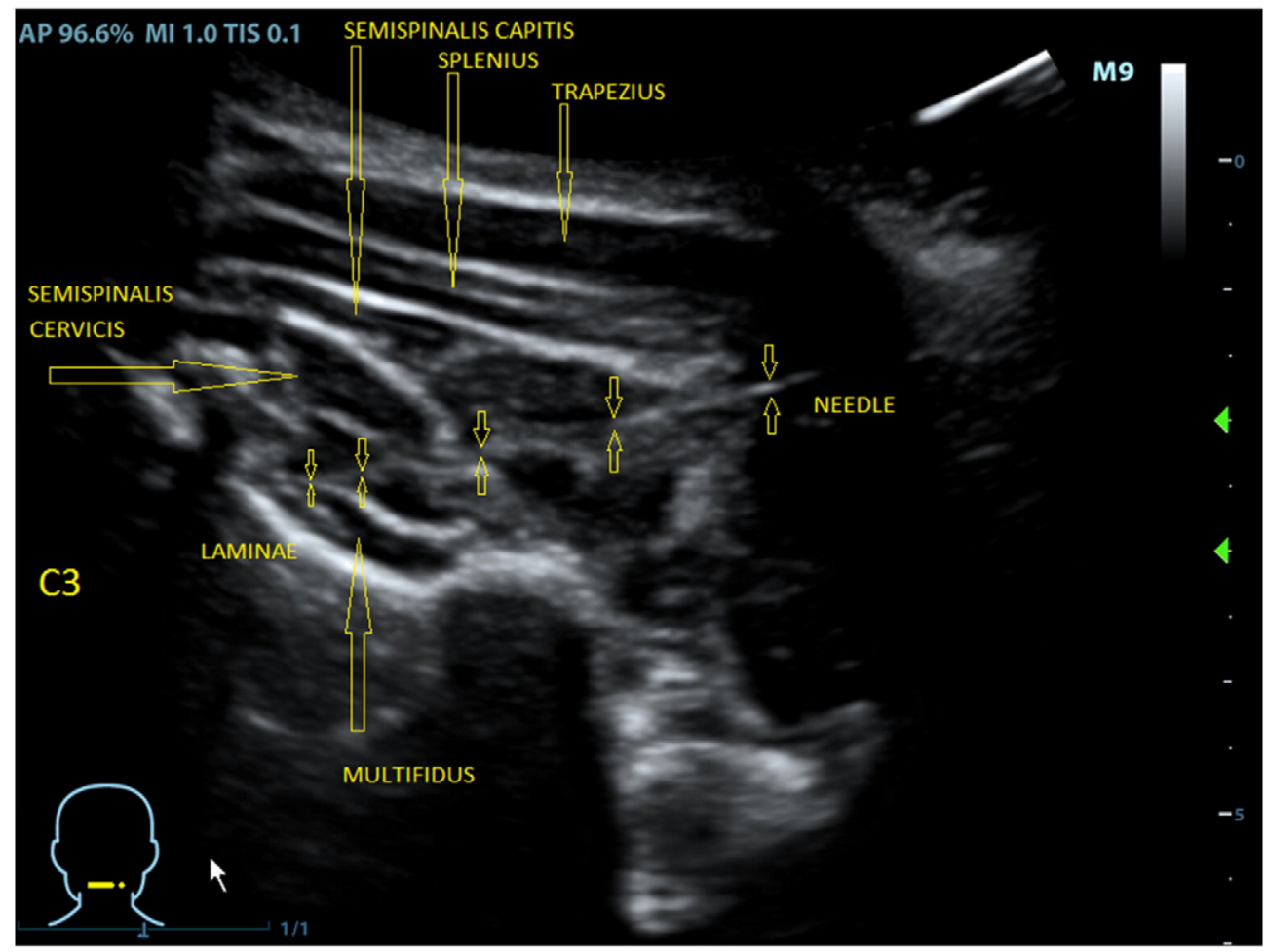

Fig 5. Ultrasound image showing dry needling of the cervical multifidus muscle at the C3-C4 level on the right side. The arrow clearly shows the needle in the deep posterior neck muscles. Color version of figure available online.

cervical multifidus after dry needling. It seems that the deep cervical extensor musculature, including the cervical multifidus, plays a major role in maintaining segmental stability. It has been suggested that activation of deep cervical extensors should be emphasized at selected spinal levels for proper management with exercise. ${ }^{7}$ Nevertheless, some authors have proposed the application of specific interventions into the muscle before starting therapeutic exercise. In such scenarios, dry needling has been proposed as a specific intervention aimed to activate the cervical multifidus muscle. ${ }^{21}$ This hypothesis is further supported by the results from a recent study in which patients with low back pain who positively responded to dry needling in the lumbar multifidus muscle exhibited an increased contracted thickness of the muscle, suggesting that dry needling was able to facilitate muscle contraction. ${ }^{13}$ This study would support clinical observation that multifidus dry needling may be applied before starting any therapeutic exercise program to more fully remedy impaired muscle contraction. ${ }^{13}$ In such a scenario, our study further confirms that the cervical multifidus muscle can be properly reached with the proposed needling approach.

The present study describes a commonly advocated needling technique for cervical multifidus. If performed properly, the potential risk inherent to this needling approach appears to be low, both in the clinical experience of the authors and as also suggested by the present study, because no adverse event was observed during any insertion. However, some precautions should be considered. For example, clinicians should avoid needling medially to minimize the possibility of penetrating the structures within the spinal canal, although this is most unlikely because of the small intervertebral space in the neck, and risk is minimized by the slight inferior angle during insertion. Clinicians should also avoid needling lateral to the vertebrae because a deep insertion could potentially strike a nerve root as it exits the intervertebral foramina. Furthermore, because the multifidus is the deepest cervical muscle, clinicians should consider that the needle also crosses other superficial muscles, such as the splenius or semispinalis, before properly reaching the multifidus. ${ }^{22}$

Finally, although this study confirmed the accuracy of treating the cervical multifidus muscle with dry needling, we should recognize some limitations. First, the dissection was conducted in 2 male cadavers and 5 patients with mechanical neck pain. Therefore, extrapolation of the current results should be considered with caution. Second, this paper does not determine the clinical effectiveness of needling of cervical multifidus. Future randomized controlled trials including dry needling of the cervical multifidus muscles are needed to determine the clinical effectiveness of this approach. Third, we did not specifically measured distance of the tip of the needle to any vascular or sensitive structure of the cervical spine to determine the real risk of the procedure. 


\section{CONCLUSION}

This cadaveric and ultrasound imaging study supports that dry needling of cervical multifidus muscle may be conducted with steel needles of $40 \mathrm{~mm}$ in length.

\section{Funding SOURCES AND CONFLICTS OF INTEREST}

No funding sources or conflicts of interest were reported for this study.

\section{CONTRIBUTORSHIP INFORMATION}

Concept development (provided idea for the research): C.F.-P., J.M.-J., J.P.-M., S.K., S.F.-C.

Design (planned the methods to generate the results): C.F.-P., J.M.-J., J.P.-M., S.K., S.F.-C.

Supervision (provided oversight, responsible for organization and implementation, writing of the manuscript): S.F.-C.

Data collection/processing (responsible for experiments, patient management, organization, or reporting data): C.F.-P., J.M.-J., J.P.-M., S.K., S.F.-C.

Analysis/interpretation (responsible for statistical analysis, evaluation, and presentation of the results): C.F.-P., J.M.-J.

Literature search (performed the literature search): C.F.-P., J.M.-J., J.P.-M., S.K., S.F.-C.

Writing (responsible for writing a substantive part of the manuscript): C.F.-P., J.M.-J., J.P.-M., S.K., S.F.-C.

Critical review (revised manuscript for intellectual content, this does not relate to spelling and grammar checking): C.F.-P., J.M.-J., J.P.-M., S.K., S.F.-C.

\section{Practical Applications}

- This study confirms the anatomic and ultrasonographic validation of needling insertion in the cervical multifidus.

- The needle does pierce the cervical multifidus during the insertion, and the tip of the needle then rests properly against the vertebral laminae.

- Dry needling of the cervical multifidus may be accurately conducted in clinical practice.

\section{REFERENCES}

1. Hoy D, March L, Woolf A, et al. The global burden of neck pain: estimates from the global burden of disease 2010 study. Ann Rheum Dis. 2014;73(7):1309-1315.

2. Jull G, Sterling M, Falla D, Treleaven J, O'Leary S. Whiplash, headache, and neck pain: research-based directions for physical therapies: research-based directions for physical therapies. Edinburgh, UK: Churchill Livingstone Elsevier; 2008.
3. O'Leary S, Falla D, Elliott JM, Jull G. Muscle dysfunction in cervical spine pain: implications for assessment and management. J Orthop Sports Phys Ther. 2009;39(5):324-333.

4. Elliott JM, Jull G, Noteboom JT, Darnell R, Galloway G, Gibbon WW. Fatty infiltration in the cervical extensor muscles in persistent whiplash-associated disorders: a magnetic resonance imaging analysis. Spine. 2006;31:847-855.

5. O'Leary S, Cagnie B, Reeve A, Jull G, Elliott JM. Is there altered activity of the extensor muscles in chronic mechanical neck pain? A functional magnetic resonance imaging study. Arch Phys Med Rehabil. 2011;92(6):929-934.

6. Fernández-de-las-Peñas C, Albert-Sanchís JC, Buil M, Benitez J, Alburquerque-Sendín F. Cross-sectional area of cervical multifidus muscle in females with chronic bilateral neck pain compared to controls. J Orthop Sports Phys Ther. 2008;38(4):175-180.

7. Schomacher J, Falla D. Function and structure of the deep cervical extensor muscles in patients with neck pain. Man Ther. 2013;18(5):360-366.

8. Vincent K, Maigne JY, Fischhoff C, Lanlo O, Dagenais S. Systematic review of manual therapies for nonspecific neck pain. Joint Bone Spine. 2013;80(5):508-515.

9. Anderson JS, Hsu AW, Vasavada AN. Morphology, architecture, and biomechanics of human cervical multifidus. Spine. 2005;30(4):86-91.

10. Dommerholt J, Fernandez-de-las Peñas C. Trigger point dry needling: an evidence and clinical-based approach. London, UK: Churchill Livingstone Elsevier; 2013.

11. Rainey $\mathrm{CE}$. The use of trigger point dry needling and intramuscular electrical stimulation for a subject with chronic low back pain: a case report. Sports Phys Ther. 2013;8(2):145-161.

12. Rock JM, Rainey CE. Treatment of nonspecific thoracic spine pain with trigger point dry needling and intramuscular electrical stimulation: a case series. Sports Phys Ther. 2014;9(5):699-711.

13. Koppenhaver SL, Walker MJ, Su J, et al. Changes in lumbar multifidus muscle function and nociceptive sensitivity in low back pain patient responders versus non-responders after dry needling treatment. Man Ther. 2015;20(6):769-776.

14. Ga H, Choi JH, Park CH, Yoon HJ. Dry needling of trigger points with and without paraspinal needling in myofascial pain syndromes in elderly patients. $J$ Altern Complement Med. 2007;13(6):617-624.

15. Lee JH, Lee H, Jo DJ. An acute cervical epidural hematoma as a complication of dry needling. Spine. 2011;36(13):E891-E893.

16. Baima J, Isaac Z. Clean versus sterile technique for common joint injections: a review from the physiatry perspective. Curr Rev Muscoskelet Med. 2007;1(2):88-91.

17. McDaniels A, Pittman D, Cotter A. Recommendations for best needling practices with respect to skin preparation. Med Acupunt. 2012;24(2):67.

18. Mayoux-Benhamou MA, Wybier M, Revel M. Strength and cross-sectional area of the dorsal neck muscles. Ergonomics. 1989;32(5):513-518.

19. Rankin G, Stokes M, Newham DJ. Size and shape of the posterior neck muscles measured by ultrasound imaging: normal values in male and females of different ages. Man Ther. 2005;10(2):108-115.

20. Kristjansson E. Reliability of ultrasonography for the cervical multifidus muscle in asymptomatic and symptomatic subjects. Man Ther. 2004;9(2):83-88.

21. Dommerholt J. Dry needling: peripheral and central considerations. J Man Manip Ther. 2011;19(4):223-227.

22. Wegley RS, Rumore AJ. Posterior cervical paraspinal musculature morphology: a cadaveric and CT scan study. $J$ Orthop Sports Phys Ther. 1986;8(1):15-26. 